\title{
20
}

\section{Investigation of Rapid Filling of Empty Pipes}

\author{
Mokhtar Guizani, Jose G. Vasconcelos, Steven J. Wright \\ and Khlifa Maalel.
}

Water mains are periodically subjected to maintenance, in which drain and refill of pipeline sections is a required practice. There is the possibility of damage to the water main during such operations, even when air relief valves and vacuum breakers are properly placed or the drain and refill are conducted slowly. Current research on this and related applications has resulted in different numerical models to simulate the rapid filling process (Liou and Hunt, 1996; Izquierdo et al. 1999) based on the assumption that a vertical interface can represent the water inflow front. Models to simulate the rapid filling of stormwater sewers have also used this assumption, as exemplified by Zhou et al. (2002). Research on the rapid filling of initially empty pipes was conducted at the National School of Engineering of Tunis (Tunisia) and the University of Michigan. This research was conducted to gain a better understanding of the filling process in closed pipes and thus try to optimize the filling operations and minimize the risk of damages. An experimental investigation was performed to characterize the inflow front and assess the validity of the vertical front assumption. In these experiments, the upstream reservoir head and the pipe slope were varied, and the inflow front was measured and characterized with the aid of digital camcorders. A piezo-resistive pressure transducer was also used to record the pressure during the filling process. Among other findings, this investigation

Guizani, M., J. Vasconcelos, S.J. Wright and K. Maalel. 2006. "Investigation of Rapid Filling of Empty Pipes." Journal of Water Management Modeling R225-20. doi: 10.14796/JWMM.R225-20.

(C) CHI 2006 www.chijournal.org ISSN: 2292-6062 (Formerly in Intelligent Modeling of Urban Water Systems. ISBN: 0-9736716-2-9) 
demonstrated that inflow fronts do not always close the pipe cross-section, invalidating the vertical interface assumption even when proposed criteria for this assumption to be applicable have been satisfied. Two different numerical models, based on the rigid column analysis proposed by Liou and Hunt (1996) and the shock-capturing technique developed by Vasconcelos et al. (2006) were used in this investigation to assess their predictive abilities in simulating the rapid filling of empty pipes. This assessment showed that while the rigid column model reproduces certain aspects of the observed flow, the shock-capturing model was capable of resolving more of the essential features of the flow.

\subsection{Introduction}

The rapid filling of pipelines creates the potential for problems caused by the entrapment of air pockets within the pipes. These include water hammer caused by rapid expulsion of air pockets (Wylie and Streeter 1993) and pressure surges. Different numerical models have been developed to describe the filling process, but their general applicability to the problem has not been demonstrated in an experimental investigation.

Several of these numerical models are based on rigid column theory including the models proposed by Wiggert (1972), Hamam and McCorquodale (1982), Li and McCorquodale (1999), Zhou et al. (2002), and Vasconcelos and Wright (2003). This approach, solving ordinary differential equations to represent the inflow advance, is based on several assumptions that include well-defined air-water interfaces and the validity of the lumped inertia formulation. An important consequence of the first assumption is that the inflow necessarily generates a distinct front that fills the pipe cross section. Two alternative criteria have been suggested as necessary to ensure a sharp front formation. The first criterion is based on the conveyance capacity of the pipe. Pressurized flow can't occur if the inflow is too small since a free surface flow will be capable of transmitting the flow without reaching the pipe crown. The second criterion, suggested by Liou and Hunt (1996), suggests that the pipe will flow full so long as the celerity of an intruding air cavity is less the advance velocity in the filled portion of the pipe. The intrusion velocity is estimated based on theory derived by Benjamin (1968). Zukoski (1966) determined that the speed of air pockets in horizontal circular pipes is given by $0.54(\mathrm{gD})^{0.5}$ with $\mathrm{D}$ the pipe diameter. Thus, air intrusion should not occur so long as the water velocity exceeds this value. Liou and Hunt (1996) presented a numerical model based on the 
rigid column assumption to describe the filling of a variable grade pipeline. To justify the assumption of a vertical front, the authors used the air intrusion velocity based criteria, but no experimental evidence was provided to assess the validity of this criterion. Other numerical models to describe the filling of pipelines, such as the one by Izquierdo et al. (1999), also assume a vertical advancing water front without discussion of flow conditions that may be required for their models to be valid.

There is reason to doubt the validity of the air intrusion velocity criterion as a basis for justifying the vertical front assumption. Although we have not been able to find previous experimental studies that address this situation directly, there have been numerous studies of the (presumed analogous and more easily observed) phenomenon of salt water intrusions into fresh water. The advantage of examining salinity intrusions is that the smaller density difference between fluids causes the intrusions to propagate more slowly. A key observation (e.g. Paez-Rivadeneira, 1997) is that a distinct front only forms when the intruding fluid actually advances into the receiving fluid. If this condition is not satisfied, for example, in the case of upstream salinity intrusion into rivers (Keulegan, 1966), a salt water wedge, controlled by interfacial shear is formed and the Benjamin analysis does not apply. The same circumstances should apply to the situation where water is displacing air in the direction of the advancing water front.

This chapter presents the results of an experimental study of the behavior of an air-water front during a rapid filling process of a pipeline generated by a sudden opening of an upstream valve. Experimental conditions were established such that the water velocity should exceed the air intrusion velocity estimated by the results of Zukoski (1966) for at least a portion of the front advance. The investigation focused on the details of the water front. The primary objective of this chapter is to assess the validity of the vertical front interface assumption, but we also compare the experimental results with predictions of numerical models proposed by Liou and Hunt (1996) and Vasconcelos et al. (2006).

\subsection{Experimental Program}

\subsubsection{Experimental Apparatus}

The experimental apparatus, indicated schematically in Figure 20.1, constructed in the hydraulics laboratory of the University of Michigan 
consists of an upstream tank (2.96 $\mathrm{m}^{2}$ in cross-sectional area) connected to an acrylic pipeline (inner diameter $\mathrm{D}=94 \mathrm{~mm}$ ), for which the slope is adjustable. This upstream tank ensures a nearly constant head during the pipe filling process. The pipe is $14.6 \mathrm{~m}$ long and was maintained in an initially empty condition. A $19 \mathrm{~cm}$-diameter riser was installed at the downstream end of the pipeline to contain the inflowing fluid. Water flow was initiated from the upstream tank by suddenly removing a plug from the upstream end of the pipe. Experiments at different upstream heads were conducted; these heads were selected such that a rigid column analysis would predict a water advance velocity in excess of the air intrusion velocity obtained from the experimental results of Zukoski (1966). For the given pipe diameter, $0.54(\mathrm{gD})^{0.5}=0.518 \mathrm{~m} / \mathrm{s}$.

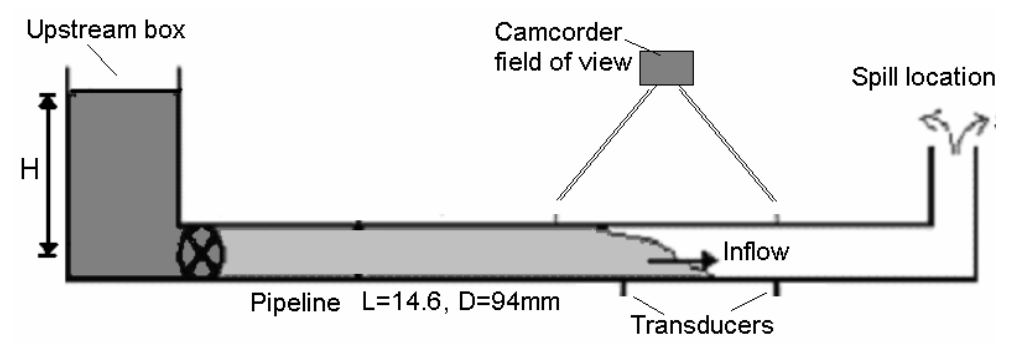

Figure 20.1 Sketch of the experimental set-up.

A high frequency response $(1000 \mathrm{~Hz})$ strain gauge pressure transducer was installed at the bottom of the pipe $12.6 \mathrm{~m}$ from the upstream tank. The output from the pressure transducer was sampled at a high frequency and recorded with a computer controlled data acquisition system.

Two digital video cameras were used to monitor and record events in the transparent pipe. Each one was positioned to provide a field of view $1 \mathrm{~m}$ wide. They were designed to record the passage of the advancing water front over a length of slightly over $2 \mathrm{~m}$ (between about 10.4 and $12.6 \mathrm{~m}$ ) since preliminary experiments indicated that this length of channel would be required to resolve the front. The recording rate was 30 frames per second for each camera. At this spatial resolution, the image quality was somewhat marginal due in part to the presence of air bubbles at the front, making the determination of the exact air-water interface difficult to determine. The error in the longitudinal measurement of the front was estimated to be $0.05 \mathrm{~m}$. For upward and downward slopes, only one camera was used and the event was recorded in two separate events. The camera was stationed to 
one side of the recording location and three repetitions were performed for each experimental condition. A second set of repetitions was then made to cover the second half of the recording interval.

\subsubsection{Experimental Variables}

Two different experimental parameters were varied in the experiments. The first one was the pipeline slope since the conveyance of the pipe should vary with slope. Three different slopes were used: horizontal, upward $0.40 \%$ and downward $1.02 \%$. The second experimental variable was the upstream pressure head in the supply tank. Three different upstream heads were investigated; in total, nine different conditions were tested and they are summarized in Table 20.1. The upstream pressure head is defined relative to the invert of the pipe at the inlet. In each experiment, the shape of the advancing front was recorded with the digital video camera(s) at a given downstream location. In addition, the pressure transducer was used to record the pressure as the front advanced.

Table 20.1 Range of experimental test conditions.

\begin{tabular}{cc}
\hline Parameter & Range tested \\
\hline Pipeline slope & Horizontal, $1.02 \%$ downward, \\
Upstream pressure head & $0.4 \%$ upward \\
& $3.9,6.6$ and 9.2 times \\
pipe diameter \\
\hline
\end{tabular}

\subsubsection{Experimental procedure}

Generally, the experimental procedure was the following:

1. the pipe was set at the desired slope by adjusting the slope of the flume in which it was installed;

2. the plug isolating the pipeline from the head tank was closed and the head tank was filled to the desired level;

3 . the digital camera(s) and the pressure transducer were started prior to the flow start;

4. the flow was started by pulling the plug, initiating the pipeline filling process; and 
5. each experimental condition was repeated three times to ensure consistent results among the runs.

\subsection{Experimental Results}

\subsubsection{General Observations}

A number of consistent observations were made over the range of experimental conditions. Although the flow was highly turbulent at the flow initiation, there was no obvious tendency for the advancing front to approach a condition with a vertical face. In fact, the length of the front increased with propagation distance down the channel. The length of the front also increases as the upstream head was decreased. Finally, the length of the front increased as the slope was altered from an adverse to a positive slope. The following sections provide the details of these observations. One additional relevant observation is that for the downward sloping channel at $\mathrm{H} / \mathrm{D}=3.9$, the pipe was only flowing full for the first few $\mathrm{cm}$ of its length while the downstream portion was flowing in the free surface flow regime.

\subsubsection{Front Shape as Function of H/D for Horizontal Slope}

The objective of this section is to characterize the shape of the inflow front for different values of $\mathrm{H} / \mathrm{D}$ and horizontal bed slope. To characterize this front shape; a simplistic approach was proposed, in which the front shape was represented using three points. The first one is the location of the front at the pipe bottom, the second is where the front depth equals half of the pipe diameter (center feature), and the third is where the front touches the pipe crown. Two digital cameras recorded the passage of the front and the evolution of the three front features with time was extracted from the video records; the results are presented in Figure 20.2.

Table 20.2 presents the front length and the first arrival time of water at the $12.6 \mathrm{~m}$ location as a function of $\mathrm{H} / \mathrm{D}$ for the horizontal slope experiments. These results were computed from the pressure transducer outputs by recording the difference between the first arrival time and the time at which the pressure head on the bottom transducer increased to a value equal to the pipe diameter. This time difference is multiplied by the computed front speed as described in more detail below. The results for the largest upstream pressure head $(\mathrm{H} / \mathrm{D}=9.2)$, show that the front width was 6.8 diameters, 
increasing slightly to 8.7 diameters for $\mathrm{H} / \mathrm{D}=6.6$, but for $\mathrm{H} / \mathrm{D}=3.9$, the front length exceeds 23 diameters and could not be captured within the field of view of the video cameras. The length could not be estimated from the pressure transducer since the reflection of the front from the downstream end of the pipeline arrived back at the measurement location prior to the pipe being filled from the upstream end of the system.
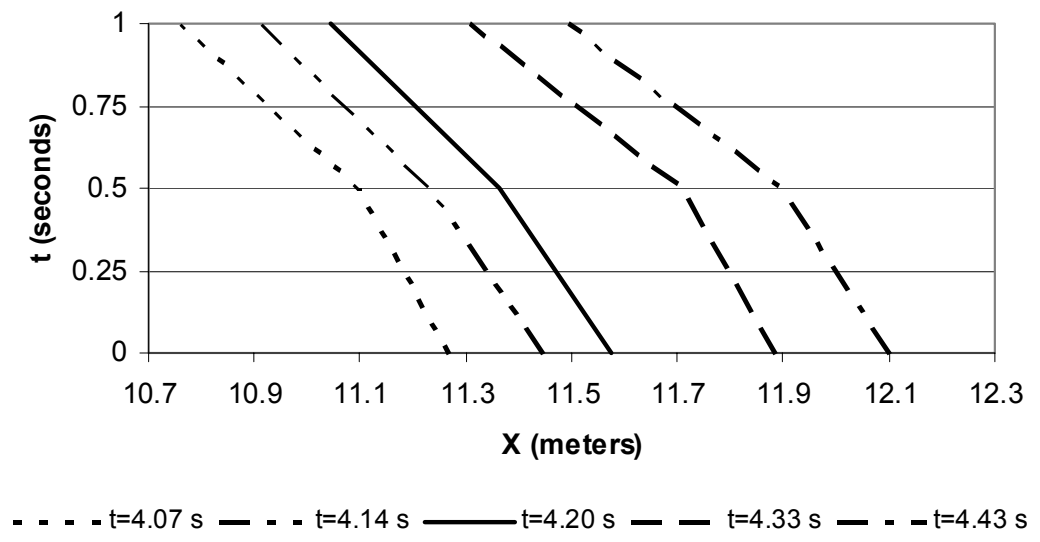

Figure 20.2 Shape of the front advance.

Table 20.2 Front length as a function of $H / D$ at $12.6 \mathrm{~m}$ station.

\begin{tabular}{lccc}
\hline & H/D & $\begin{array}{c}\text { First Arrival time } \\
\text { of water (s) }\end{array}$ & $\begin{array}{c}\text { Normalized } \\
\text { Front length } \\
\text { (L/D) }\end{array}$ \\
\hline Horizontal slope & 9.2 & 4.4 & 6.8 \\
& 6.6 & 5.2 & 8.7 \\
& 3.9 & 6.6 & $>23$ \\
\hline
\end{tabular}

\subsubsection{Trajectory of Filling Front}

Since the video camera records the front location at a 30 frame per second rate, it is possible to estimate the propagation speed of the front as it passes through the recording section. This was done for the bottom, center and top 
portions of the front (with the exception of the $\mathrm{H} / \mathrm{D}=3.9$ experiments where the filling of the top of the pipe could not be observed.) The locations (averages from the three repetitions) of the various front features were estimated every few frames and these are plotted in Figure 20.3 for the horizontal slope experiments. The trajectories indicate a fairly constant slope (indicating constant front velocity) across the recording location. The slope (actually the inverse of the slope) for each of the trajectories such as plotted in Figure 20.3 was computed to determine the propagation speed for the various portions of the advancing front. The speed of propagation of the bottom, center and top of the front for each of the different H/D values and for each of the three slopes is presented in Table 20.3. The results presented in this table can be used to obtain several important conclusions. First of all, every one of the frontal speeds considerably exceeded the critical "intrusion" velocity of $0.52 \mathrm{~m} / \mathrm{s}$ as defined by Liou and Hunt (1996). According to their criteria, each one of the experiments should have experienced a vertical front and this is clearly not the case as indicated in the results in Figure 20.2 and Table 20.2. A second observation is that in all but two cases, the bottom of the front is indicated to be propagating faster than the top so that the front length would increase with time or distance down the channel; this is also consistent with visual observations of the fronts. Although there are some inconsistencies in this general trend, this is probably related to the accuracy of the data from which these trajectories were generated. The most accurate results should have been for the horizontal slope experiments since these were determined from simultaneous images with the two video cameras, whereas the other two slope cases involved two successive experiments with one video camera and more potential for the introduction of measurement error. A final observation is that the front speed is higher for higher $\mathrm{H} / \mathrm{D}$ values, as would be expected. The pipe slope however did not have a major impact on front propagation speed with the possible exception of the $\mathrm{H} / \mathrm{D}=3.9$ case for which the downward sloping pipe tended to have a faster propagating front. This result is consistent with the observation that the pipe did not flow full for this experiment, requiring a faster front velocity for a given driving head.

Although this study was originally planned to investigate the validity of the air intrusion criterion for the vertical front assumption, it is possible to draw some tentative conclusions regarding the ability of a conveyance criterion to describe the ability of a pipe to flow full. Most analyses consider that the effects of wall shear can be incorporated into numerical models by means of a quasi-steady analysis in which steady state friction factors can be used in conjunction with local velocities to describe local shear forces. 
While this assumption may not be strictly valid in the immediate vicinity of the advancing front or at the initiation of flow where the front is rapidly evolving, it appears to otherwise be reasonable. If this approach is used, it can be inferred that for some combinations of flow velocity and pipeline geometry (i.e. slope) a pipeline will not flow full simply because it is capable of conveying the flow in a free surface state.

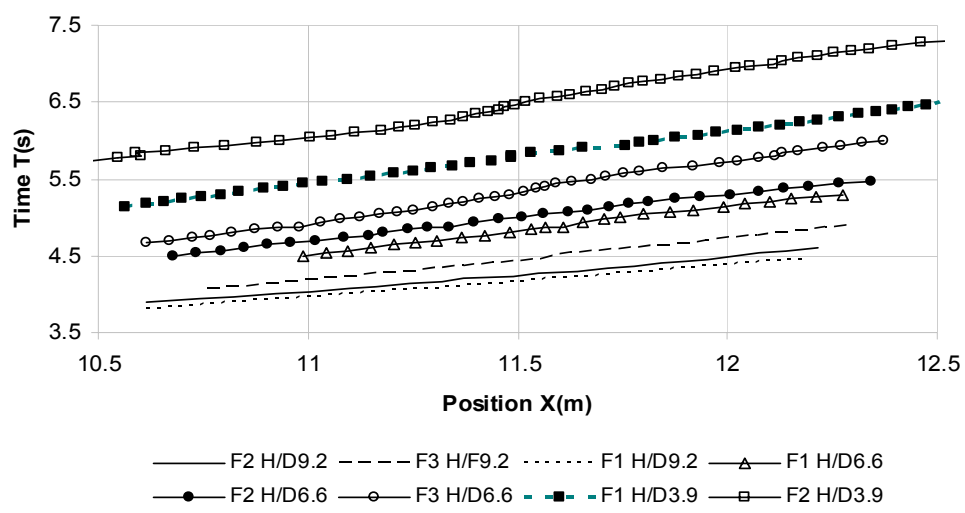

Figure 20.3 Front trajectories for the horizontal slope experiments (legend key : $F=$ feature numbers $H / D=H / D$ ratio).

It is not straightforward to assess the conveyance capability for a given flow configuration since the energy slope must be used in the hydraulic resistance relation as opposed to the actual pipe slope. However, we can use the results in Table 20.3 for the downward sloping pipe with a local energy slope between the reservoir and the measurement location; for simplicity reasons, this was performed neglecting any entrance loss into the pipeline.

This slope was used in the Manning equation (assuming full pipe flow) with a Manning coefficient of 0.010 . For the reservoir heads, $\mathrm{H} / \mathrm{D}$, of 9.2, 6.6, and 3.9, the required velocities for full pipe flow are 1.95, 1.66, and 1.28 $\mathrm{m} / \mathrm{s}$, respectively. The front velocity for the same three conditions is computed from Table 20.3 as $2.0,1,67$, and $1.60 \mathrm{~m} / \mathrm{s}$ by taking the average velocity given in the table for the three front features. These results indicate that the two higher heads required a pressurized flow to convey the flow admitted into the pipeline while the low head case did not. This is also consistent with visual observations of the different flow conditions where the lower head resulted in a free surface flow except in a very limited region 
near the pipe entrance; this occurrence may have been related to the specific inlet conditions. Another implication of this analysis is that if the pipeline were sufficiently long, all of the cases with the downward sloping pipe would have eventually propagated with a free surface flow at the front since the pipe slope was sufficient to prevent a full pipe condition at uniform flow conditions.

Table 20.3 Speeds of different front features $(\mathrm{m} / \mathrm{s})$.

\begin{tabular}{lccc}
\hline \multicolumn{1}{r}{ H/D } & 9.2 & 6.6 & 3.9 \\
\hline Bottom & 2.3 & 1.8 & 1.4 \\
Center & 2.2 & 1.8 & 1.4 \\
Top & 1.9 & 1.4 & -- \\
& \multicolumn{3}{c}{ Horizontal slope } \\
Bottom & 2.2 & 1.6 & 1.3 \\
Center & 2.3 & 2.2 & 1.6 \\
Top & 1.9 & 1.6 & 1.5 \\
& \multicolumn{3}{c}{ Downward slope } \\
Bottom & 2.3 & 1.8 & 1.6 \\
Center & 2.2 & 1.7 & 1.6 \\
Top & 1.5 & 1.5 & -- \\
\hline
\end{tabular}

Note that the conveyance consideration does not have any direct implication as to whether or not the advancing front is vertical. It only addresses the issue of whether the conduit flows full at some location. For example, if we consider the low head cases of $\mathrm{H} / \mathrm{D}=3.9$ but either on the horizontal or adverse slopes, it is clear that the conduit must flow full once the front has advanced a certain distance since a uniform free surface flow cannot be attained on those slopes. This implies that once the flow becomes pressurized, the location where the flow touches the pipe crown will begin to move downstream although probably at a different speed than other features of the intrusion. In these cases, it is likely that the length of the pressurization front will be considerable and the use of a vertical front assumption will be of limited utility in describing various aspects of the flow. Alternatively, a combined free surface/pressurized flow simulator such as that proposed by Vasconcelos, et al (2006) should be capable of resolving the basic features of the flow, as long as air pockets are not entrapped by the flow. 


\subsubsection{Pressure Measurements of the Inflow Front}

This section presents the results from the measurements made with a piezoresistive pressure transducer located at the bottom of the pipe at the $12.6 \mathrm{~m}$ station. The pressure head results are also compared with the depth changes observed at the same station recorded with the digital camera.

When there is no water at the recording station, the transducer head is zero. When the advancing front reaches the recording station, the pressure increases as the water level increases in the pipe. If a hydrostatic pressure distribution is assumed, the pressure can be converted directly to water depth up to the point that the pipe becomes pressurized, after which the surcharge pressure in the pipe can be obtained. We are primarily interested in the interval between the arrival of the front at the measurement location and the time that the pressurized condition is achieved. Figure 20.4 shows an example of the pressure transients recorded during an experiment with $\mathrm{H} / \mathrm{D}=$ 9.2. Three repetitions of the same experimental condition are presented, with small differences among the three runs, presumably due primarily to the intense turbulence at the front and also possibly small variations in the way that the flow was initiated. When the pressure head rises above $0.094 \mathrm{~m}$, the pipe should be full. There is an indication of a sharper front than was obtained from the analysis of the video records.

Transducer data at $X=12.6 \mathrm{~m}-\mathrm{H} / \mathrm{D}=9.2$ - Horizontal slope

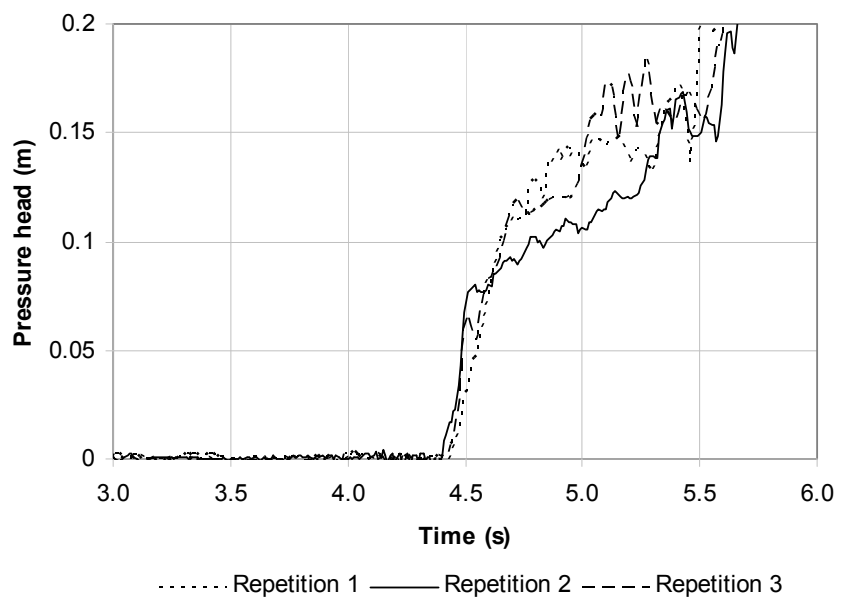

Figure 20.4 Pressure transients recorded during experiments with sudden opening of the plug at relative upstream water depth of $H / D=9.2$. 
A comparison between transducer head and depths observed in the video images is shown in Figures 20.5 and 20.6. Three repetitions from both video and transducer data are plotted for each case. The depth changes observed in the movies and the pressure transducer are generally in good agreement. There is a tendency of the measured pressure head to have a sharper rise than the depth recorded in the movies. This may be caused by the turbulence at the vicinity of the front, which has a dominant effect in that region of the flow (Whitham, 1955).

This is a clear indication that the hydrostatic assumption for that portion of the flow isn't adequate. Finally, it is interesting to notice the pressure oscillations indicated in Figure 20.6 somewhat after the passage of the front. The same data re-plotted at a different pressure scale are presented in Figure 20.7. The pressure fluctuations are due to the expulsion of discrete air bubbles at the pipe end; these have been reported in previous studies ( $\mathrm{Li}$ and McCorquodale, 1999; Vasconcelos and Wright, 2006) and are a feature of all pressure measurements in which we have seen air bubbles expelled somewhere from the piping system. A numerical model that predicts a vertical filling front would not be able to predict the occurrence of this type of phenomenon since a vertical front would not be able to trap air in the experimental configuration that were studied. Similar pressure oscillations, although less severe were also observed in the experiments with $\mathrm{H} / \mathrm{D}=6.6$.

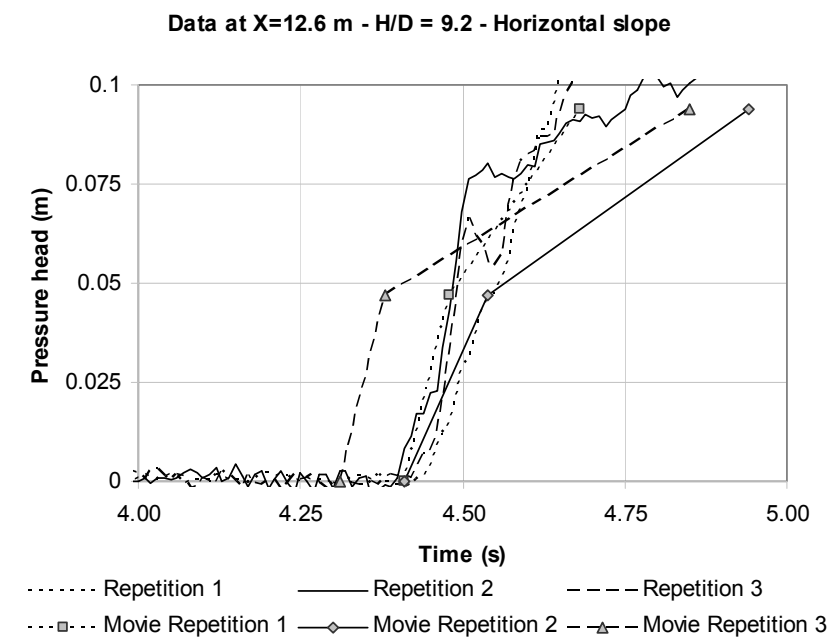

Figure 20.5 Pressure transients recorded during experiments with sudden opening of the plug at relative upstream water depth of 9.2 . 
Data at $X=12.6 m-H / D=3.9-$ Horizontal slope

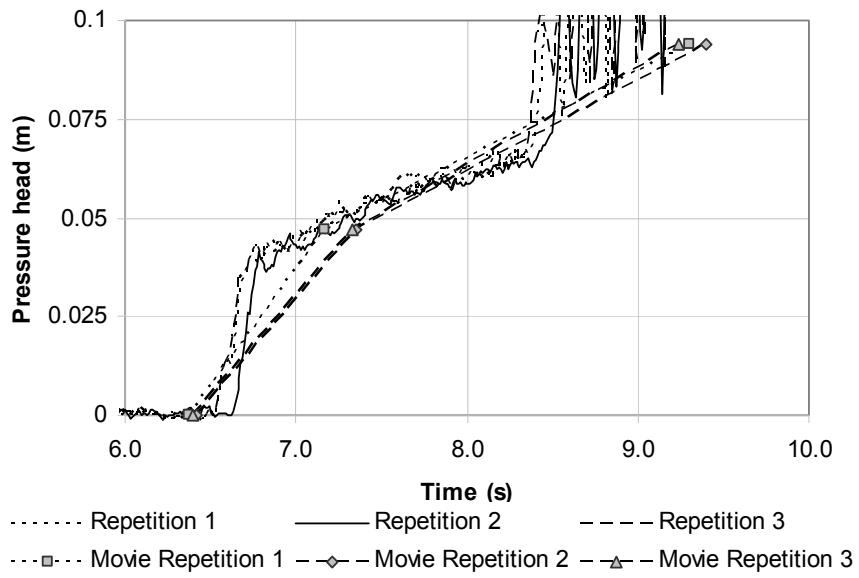

Figure 20.6 Pressure transients recorded during experiments with sudden opening of the plug at relative upstream water depth of 3.9.

\subsubsection{Numerical Computations}

Two different numerical models that simulate the rapid filling process in closed conduits, were implemented to make a comparison to observed front trajectories and pressure histories for the horizontal slope case. The first model is based on the rigid column assumption while the second is known as the Decoupled Pressure Approach (DPA) and proposed by Vasconcelos et al (2006) to simulate flow regime transition events in closed pipes. The rigid column model describes the increasing length of the column with time incorporating fluid acceleration and as well as local and friction losses; the general structure is similar to the model proposed by Liou and Hunt (1996). This DPA model implements a solution to the St. Venant equations in a fashion somewhat similar to the more familiar Preissmann Slot model so that pressurized flow can be described with free surface flow equations. In order to implement this solution, it is necessary to assume a very small initial depth in the pipeline in order that the free surface flow equations are defined prior to the arrival of the advancing front. 
Transducer data at $X=12.6 \mathrm{~m}-\mathrm{H} / \mathrm{D}=3.9-$ Horizontal slope

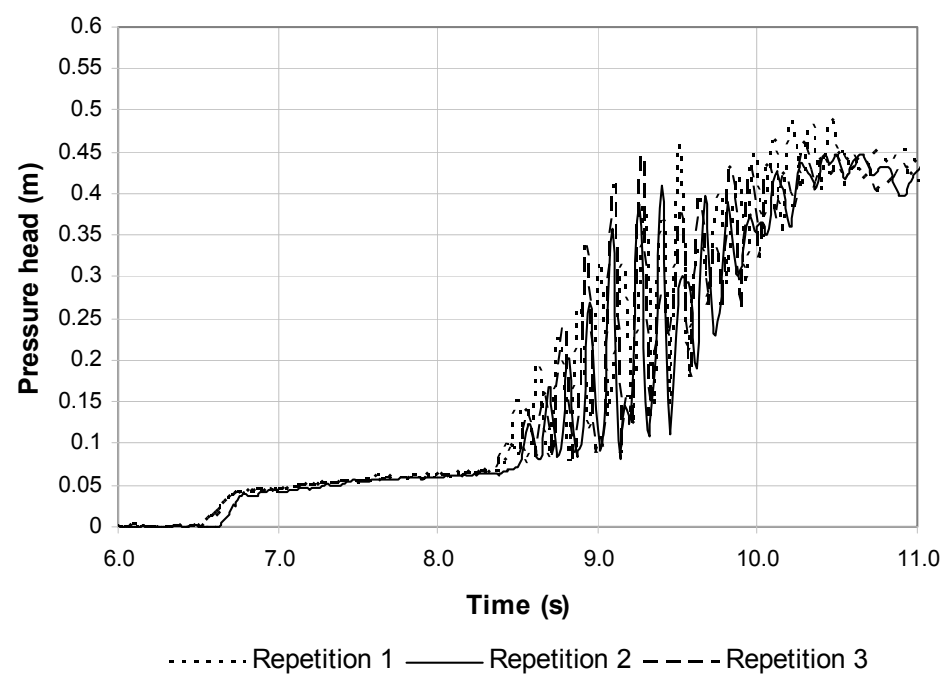

Figure 20.7 Pressure transients showing pressure fluctuations due to expulsion of air bubbles at downstream end of system.

A comparison between the front trajectory and the pressure head within the pipe at the $12.6 \mathrm{~m}$ station between the rigid column model and the experiments is shown in Figure 20.8. In order to make a comparison, a few key assumptions are required. First, it as assumed that the pressure head in the rigid column model represents the pressure at the center of the pipe; the model pressure is increased by half the pipe diameter in order to compute the bottom pressure. In addition, a Darcy-Weisbach friction factor of 0.022 was use to estimate wall shear and no entrance loss was considered at the pipe entrance although the conversion from potential energy in the reservoir to kinetic energy in the pipe was included in the model that Liou and Hunt (1996) apparently did not do. The computed results are quite sensitive to these specifications. The computed pressure increases instantaneously from zero with the arrival of a vertical front at a given location. After that, the pressure increases slowly. The model predictions are reasonably accurate for the two higher head experiments, both in terms of the timing of the front arrival and that assumes a vertical front. Anticipating system behavior in systems with more complex geometries poses even more difficulties; this suggests caution in applying rigid column models without careful 
consideration of the potential consequences of the lack of general applicability of the model assumption.

Further analysis suggests a more satisfactory criterion for ensuring that the pipeline will flow full would be based on the pipeline conveyance; additional experiments are required to confirm this observation. This criterion is not straightforward to apply as it will depend on local conditions at the front since the energy slope is required to compute the pipeline conveyance. Application of this criterion to the experiments indicates that it can distinguish conditions in which a pipeline will flow full, but alone cannot ensure a narrow length of the advancing front. The experiments were performed in a relatively short length of pipeline (approximately 150 diameters, limited by laboratory constraints) and most applications would involve much longer pipelines. Since the experimental results indicate that the front length grows with distance, the implication is that the rigid column model may be less than satisfactory in longer pipelines. the subsequent pressure increase. Since the observed length of the front was fairly small (less than ten diameters) for these experiments, it seems that the rigid column model is reasonably satisfactory to compute system behavior. The agreement is much less satisfactory for the lowest head experiment in which the observed front length is much greater. The simulations were quite sensitive to the choice of hydraulic losses and a more careful determination of these would be required to draw definite conclusions.

The pressure predictions from the DPA model at the $12.6 \mathrm{~m}$ station are plotted against the experimental measurements in Figure 20.9. This model is capable of resolving the general features of the advancing front as indicated for the three cases simulated. Although there is a slight tendency to overestimate the arrival time of the front of the front, discrepancies in timing are less than about $0.25 \mathrm{~s}$. The timing is also sensitive to the choice of friction factors. There is also a tendency of the computed pressures to be smaller than the measured values, particularly for the larger H/D experiments. This is probably related to the fact that the shallow water equations neglect the vertical accelerations that apparently give larger pressures near the front than would otherwise be expected. These simulations are capable of computing the reflections off the riser at the downstream end of the pipeline and these are indicated in the plots; the timing of this phenomenon also seems to be quite good in all cases. 

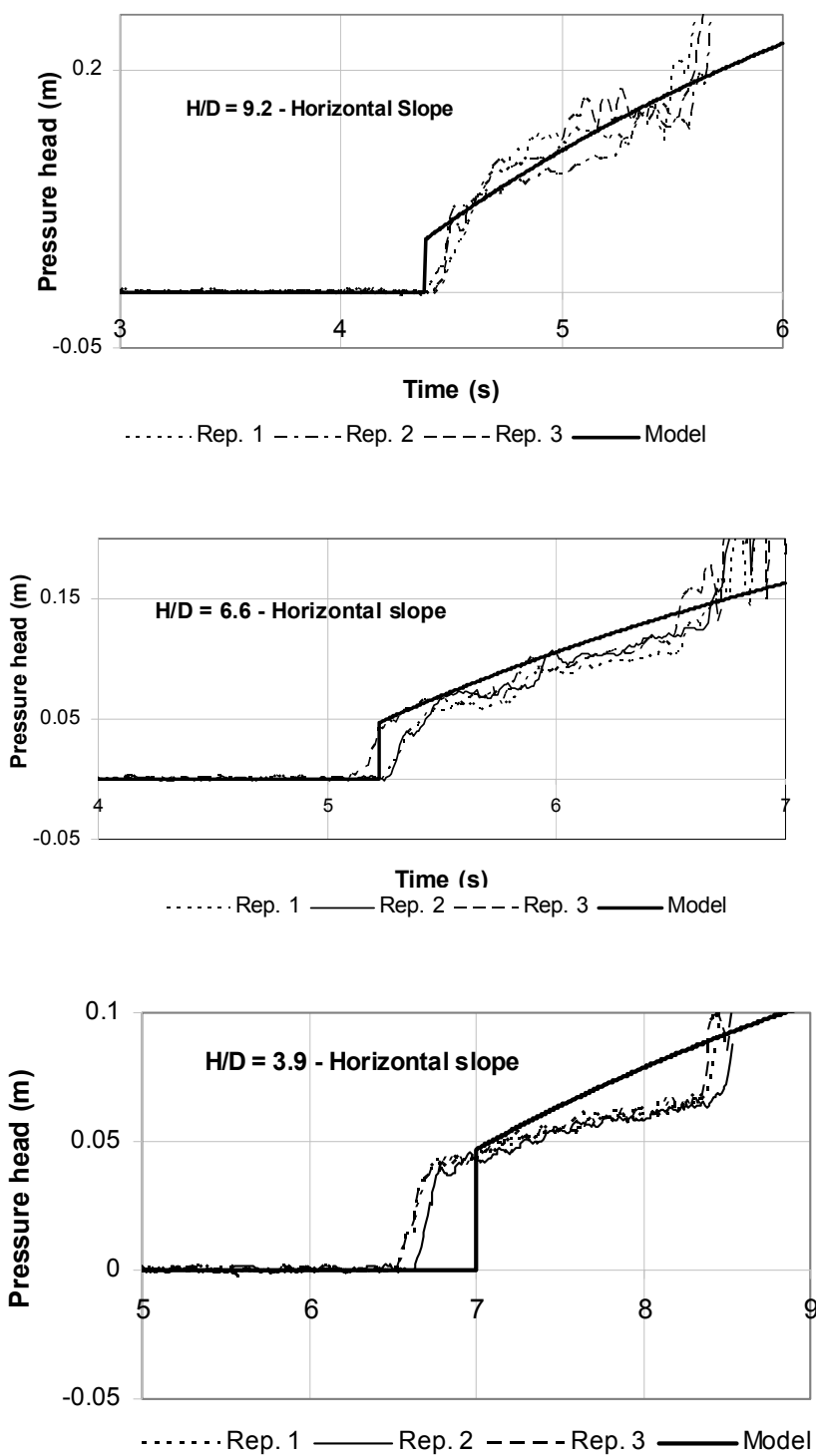

Figure 20.8 Pressure head measurements obtained with the pressure transducer at the $12.6 \mathrm{~m}$ station for horizontal slope and numerical predictions from the rigid column model. 

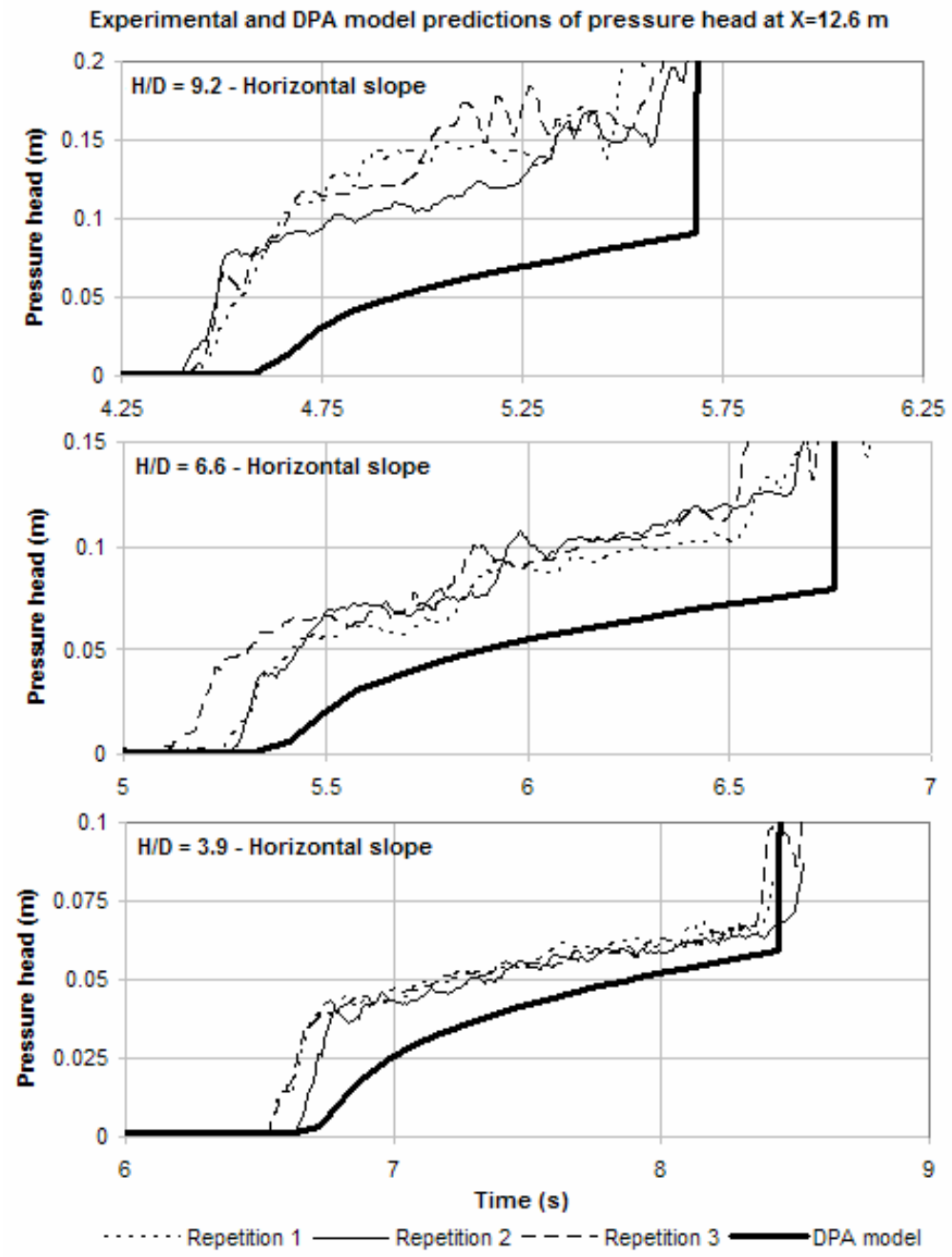

Figure 20.9 Pressure head measurements obtained with the pressure transducer at the $12.6 \mathrm{~m}$ station for horizontal slope and numerical predictions from the DPA model. 


\subsection{Summary and Conclusions}

In this chapter we have presented the results of an experimental study on the behavior of the air-water front during the rapid filling of an initially empty pipeline. The purpose of the experiments was to examine the validity of the traditional assumption of a vertical interface between air and water; this is required in order to apply rigid column numerical models. There has been little discussion of this assumption in previous studies and, to our knowledge, no previous experimental validation of the assumption. Liou and Hunt (1996) suggested a criterion based on analysis by Benjamin (1968) for air intrusion into water-filled conduits in order for the vertical front assumption to be valid. The experiments presented in this study were conducted to test this assumption. A series of experiments over a range of upstream reservoir heads and pipe slopes, all of which should have satisfied the air intrusion criterion for a vertical front were conducted and the shape of the front was measured by visually analyzing digital video records of the experiments and by measuring the bottom pressure in the pipe as the front passed the measurement location.

The experiments all demonstrated a frontal shape that is more like an advancing dam-break front even when the advance velocity was several times larger than the limiting velocity suggested by the air intrusion criterion. Not only were the fronts not vertical, but the lengths also increased with propagation distance down the channel. In one specific experiment, the pipe did not flow full as the experimental conditions allowed the passage of the transient as a free surface flow.

The significance of the vertical front approximation relates to the possibility of air entrapment within a rapidly filling pipeline and the potential for operational problems to develop when this air is expelled from the system. In the simple experimental system that was developed for this study, a near-vertical advancing front would expel the air ahead of it and there would be no influence by the air on the system performance. In the experiments performed, the filling of the pipeline occurred in at least some cases in a manner in which significant air was trapped and significant pressure spikes were recorded (see Figure 20.7 for example). This occurrence would not be anticipated in an analysis that assumes a vertical front. Anticipating system behavior in systems with more complex geometries poses even more difficulties; this suggests caution in applying rigid column models without careful consideration of the potential consequences of the lack of general applicability of the model assumption. 
Two numerical models, one utilizing the rigid column assumption and another that solves for the combined free surface-pressurized flow were applied to simulate the experimental data. It was found that the rigid column data generally performed reasonably well in predicting the observed pressure history so long as the observed front length was not too large, but the agreement became less satisfactory for flow conditions in which the front length was significant. The DPA model proposed by Vasconcelos, et al (2006) is capable of resolving the timing and the general shape of the pressurization front. It would also be able to simulate those conditions in which the pipe does not flow full.

\section{Acknowledgments}

The first author gratefully acknowledges Prof. Steven J. Wright for hosting Guizani Mokhtar at the Laboratory of hydraulics and civil engineering at the University of Michigan in order to perform the experimental investigation. The authors acknowledge the support of LMHE, The Hydraulic and Environment laboratory, which has provided a fellowship to support the first author. Also, the authors would like to acknowledge the support of $\mathrm{CNPq}$, a Brazilian Government entity committed to the development of science and technology, which has provided a fellowship to support the $\mathrm{PhD}$ studies of the second author.

\section{References}

Benjamin, T. B. (1968). "Gravity currents and related phenomena." J. Fluid Mech., 31(2), 209-248.

Hamam, M. A. and McCorquodale, J. A. (1982). "Modeling mixed flow in storm sewers." Ca. J. Civ. Engrg., (9), 189-196.

Izquierdo, J., Fuertes, J., Cabrera, E., Iglesias, P. L., and Garcia-Serra, J. (1999). "Pipeline start-up with entrapped air." J. Hyd. Res., 37(5), 579-590.

Keulegan, G.H. (1966) "The mechanism of an arrested saline wedge." Chapter 11 in Estuarine and Coastline Hydrodynamics, A. Ippen (ed.) McGraw Hill.

Li, J. and McCorquodale, A. (1999). "Modeling mixed flow in storm sewers." J. Hydr. Engrg., 125(11), 1170-1180.

Liou, C. P. and Hunt, W. A. (1996). "Filling of pipelines with undulating elevation profiles." J. Hydr. Engrg., 122(10), 534-539.

Paez-Rivadeneira, D.L. (1997), "Density current propagation over horizontal boundaries, an experimental investigation," Ph.D. dissertation, The University of Michigan. 
Vasconcelos, J. G. and Wright, S. J. (2003). "Surges associated with air expulsion in near-horizontal pipelines." Proc. FEDSM03 - 4th ASME-JSME Joint Fluids Engrg. Conference, Honolulu, HW.ASME/JSME.

Vasconcelos, J. G., Wright, S. J., and Roe, P. L. (2006). "Decoupled pressure approach for the simulation of flow regime transition in sewers. In press, Journal of Hydraulic Engineering.

Whitham G. B. (1955) "The effects of hydraulic resistance in the dam-break problem". Proceedings of the Royal Society of London, Series A, Mathematical and Physical Sciences, Vol. 227, No. 1170, 399-407

Wiggert, D. C. (1972). "Transient flow in free-surface, pressurized systems." J. Hydr. Div., 98(HY1), 11-27.

Wylie, E. B. and Streeter, V. L. (1993). Fluid Transients in Systems. Prentice Hall, Upper Saddle River, NJ.

Zhou, F., Hicks, F. E., and Steffler, P. M. (2002). "Transient flow in a rapidly filling horizontal pipe containing trapped air." J. Hydr. Engrg., 128(6), 625-634.

Zukoski, E. E. (1966). "Influence of viscosity, surface tension, and inclination on motion of long bubbles in closed pipes." J. Fluid Mech., 25(4), 821-837. 